\title{
BRCA1 genomic deletions are major founder mutations in Dutch breast cancer patients
}

\author{
Anne Petrij-Bosch ${ }^{1}$, Tamara Peelen ${ }^{1}$, Margreethe van Vliet ${ }^{1}$, Ronald van Eijk ${ }^{2}$, Renske Olmer ${ }^{1}$, \\ Marion Drüsedau ${ }^{1}$, Frans B.L. Hogervorst ${ }^{3}$, Sandra Hageman ${ }^{3}$, Petronella J.W. Arts ${ }^{4}$, \\ Marjolijn J.L. Ligtenberg ${ }^{4}$, Hanne Meijers-Heijboer ${ }^{5}$, Jan G.M. Klijn ${ }^{6}$, Hans F.A. Vasen ${ }^{7}$, \\ Cees J. Cornelisse ${ }^{2}$, Laura J. van't Veer ${ }^{3}$, Egbert Bakker ${ }^{1}$, Gert-Jan B. van Ommen ${ }^{1}$ \& Peter Devilee ${ }^{1,2}$
}

To date, more than 300 distinct small deletions, insertions and point mutations, mostly leading to premature termination of translation ${ }^{1}$, have been reported in the breast/ovarian-cancer susceptibility gene BRCA1. The elevated frequencies of some mutations in certain ethnic subpopulations ${ }^{2-4}$ are caused by founder effects 5,6 , rather than by mutation hotspots. Here we report that the currently available mutation spectrum of BRCA1 has been biased by PCR-based mutation-screening methods, such as SSCP, the protein truncation test (PTT) and direct sequencing, using genomic DNA as template. Three large genomic deletions that are not detected by these approaches comprise $36 \%$ of all $B R C A 1$ mutations found in Dutch breastcancer families to date. A 510-bp Alu-mediated deletion comprising exon 22 was found in 8 of 170 breast-cancer families recruited for research purposes and in 6 of 49 probands referred to the Amsterdam Family Cancer Clinic for genetic counselling. In addition, a 3,835-bp Alu-mediated deletion encompassing exon 13 was detected in 6 of the 170 research families, while an deletion of approximately 14 kb was detected in a single family. Haplotype analyses indicated that each recurrent deletion had a single common ancestor.

We selected four families (RUL5, RUL105, EUR9 and EUR21) for further study because they each showed strong evidence of linkage to $B R C A 1$, although two index cases of each family had remained negative after the PTT of exon 11 (refs 4,7,8). All 22 coding exons and their immediate intron sequences were sequenced in one index case carrying the disease haplotype from each family. Other than a number of previously pub- $a$ lished exonic polymorphisms ${ }^{1}$, no conclusive mutations were found. $A \mathrm{G} \rightarrow \mathrm{A}$ mutation at position IVS22 +5 was found to co-segregate with the disease haplotype in family EUR21. This $\mathrm{G}$ is conserved in $84 \%$ of splice-acceptor sites $^{9}$, suggesting a splice-site mutation. RT-PCR analysis of exons 20-24 revealed an additional smaller band in patient RUL105.6 and, surprisingly, also in the proband of family RUL105 (Fig. 1a), in which the IVS $22+5 \mathrm{G} \rightarrow \mathrm{A}$ mutation had not been detected. In both

cases, the sequence of these products revealed a deletion of exon 22 . This deletion causes a frameshift and premature translation termination, which removes the last 60 amino acids of the protein. We conclude that in EUR21, but not in RUL105, the deletion of exon 22 from the mRNA is caused by the IVS $22+$ $5 \mathrm{G} \rightarrow \mathrm{A}$ mutation. The ratio between full-length and exon 22-deleted RT-PCR products was variable between duplicate experiments when lymphocyte RNA was used as starting material (Fig. $1 b$ ). Given the very low expression levels of $B R C A 1$ in these cells, we attribute this to coincidental preferential amplification. No alterations were detected in the RT-PCR products spanning exons 2-10 and 12-24 from patient EUR9.5 (data not shown). No RNA was available from any breast-cancer case in family RUL5, precluding RT-PCR analysis.

Two additional RT-PCR variants were found in eighteen samples derived from breast- or ovarian-cancer patients who requested genetic counselling at the Leiden Family Cancer Clinic (Fig. 1c). Patient B13 was found to contain a variant lacking exons 13-16 (801 bp), causing an in-frame deletion that removes residues $1396-1662$ of the protein. The variant in patient B20 lacked exon 13 (172 bp), causing a frameshift and a stop codon at position 1398. Sequence analysis of the intronexon borders flanking the deletions in these two patients did not reveal a change in the splice sites.

We performed Southern-blot analysis of genomic DNA derived from these patients. DNA samples were digested with either HindIII or BglII, and blotted with probes derived from

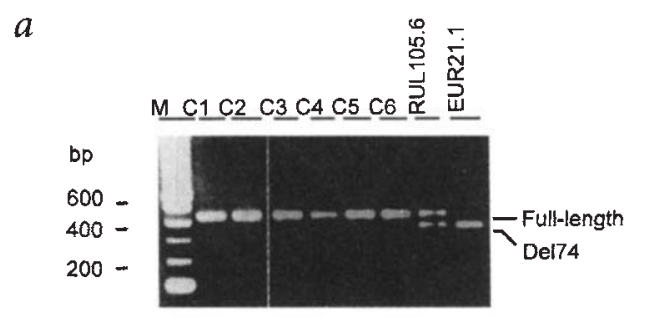

Fig. 1 RT-PCR analysis of BRCA1 mRNA isolated from fresh lymphocytes using primers spanning exons 12-24. a, C1-C6: unrelated control individuals. Dei74 denotes a fragment containing a 74-bp deletion corresponding to exon $22 . \boldsymbol{b}$, Three independent RT-PCR experiments from the same CDNA prepared from EUR21.1. $c$ Results from breast-cancer probands requesting genetic counselling. The Del172 fragment amplified in 820 represents a deletion of exon 13; the Del801 fragment amplified in B13 represents a deletion of exons 13-16. In both B13 and B20, the presence of the transcript generated from the wild-type allele was confirmed

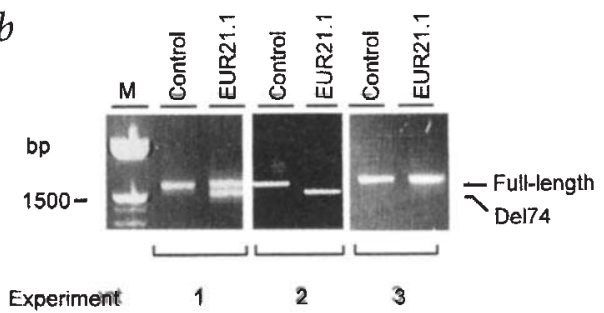

using primers which map within the region deleted in the other allele (data not shown). M, molecular-weight marker.

${ }^{1}$ Department of Human Genetics and ${ }^{2}$ Department of Pathology, Leiden University Medical Centre, P.O. Box 9600,2300 RA Leiden, The Netherlands. ${ }^{3}$ Department of Pathology and Family Cancer Clinic, The Netherlands Cancer Institute, Amsterdam, The Netherlands. ${ }^{4}$ Department of Human Genetics, University Hospital, Nijmegen, The Netherlands. ${ }^{5}$ Department of Clinical Genetics, Erasmus University, Rotterdam, The Netherlands. ${ }^{6}$ Daniel den Hoed Cancer Centre and Family Cancer Clinic, Rotterdam, The Netherlands. ${ }^{7}$ Foundation for the Detection of Hereditary Tumours, Leiden, The Netheriands. Correspondence should be addressed to P.D. $e$-mail: devilee@ruly46.medfac.leidenuniv.nl 

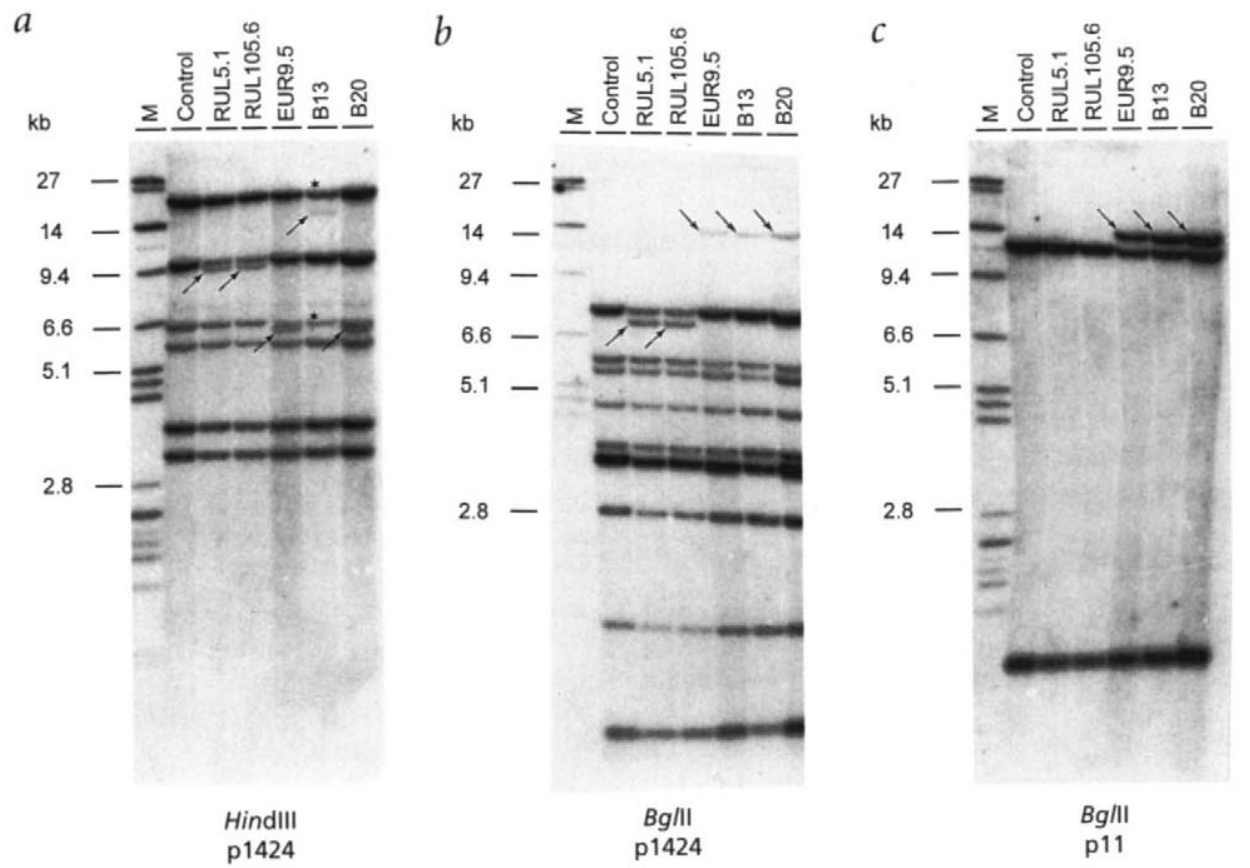

Fig. 2 Southern-blot analysis. a-c, Genomic DNA was digested and hybridized with the indicated restriction enzyme and probe. Arrows denote presumed deletion-junction fragments; asterisk denotes fragments in which a reduced signal intensity was confirmed by Phosphorimager analysis. $M$, molecularweight marker. d, Gene organization of the $3^{\prime}$ half of $B R C A 1$, to scale, and restriction maps for $B g / I I(B)$ and HindIII (H), as predicted by the genomic sequence of the $B R C A 1$ gene ${ }^{10}$. BB represents two $B g / l 1$ sites spaced $40 \mathrm{bp}$ apart. Numbers refer to size of restriction fragments (in $\mathrm{kb}$ ) as detected by probe $\mathrm{p} 11$ or $\mathrm{p} 1424$, above the bar for $B g / I$, below the bar for HindIII. The 3.6-kb Hind III fragment in parentheses becomes part of the junction fragments generated by the 3.8- and 14-kb deletions. The solid blocks below the bar represent genomic deletions, encompassing the exons indicated, which were found in patient cases.

$d$

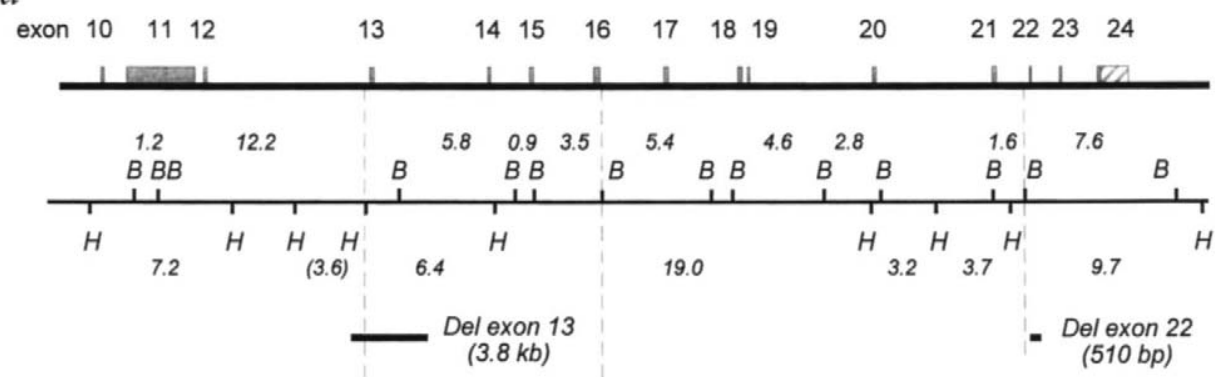

Del exons 13-16

$(\sim 14 \mathrm{~kb})$

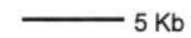

exon 11 (p11) or exons 14-24 (p1424), respectively. Patient RUL105.6 revealed aberrant bands, with both restriction enzymes (Fig. $2 a, b$ ), using pl 424 as probe, excluding the possibility of a restriction fragment-length polymorphism. Patient RUL5.1 showed identical restriction-fragment patterns, suggesting the same DNA rearrangement as in RUL105.6. Similarly, genomic DNA from patients B20 and EUR9.5 showed identical additional bands relative to the expected pattern (Fig. $2 a, c$ ), but they were different from those observed in RUL5.1 and RUL105.6 samples. Finally, patient B13 showed a 14-kb aberrant $B g l I I$ band with p 11 (Fig. $2 c$ ); this band appears to be of equal size to the one observed in B20 and EUR9.5. These bands were also faintly detected by probe p1424 (Fig. $2 b$ ). However, the HindIII patterns of patients EUR9.5, B13 and B20, obtained using p1424 (Fig. 2a), indicated that patient B13 lacked the rearranged band of about $6.3 \mathrm{~kb}$ seen in EUR9.5 and B20, while showing a reduced intensity of the constitutional 6.4- and 19-kb bands, containing exons 13-14 and 15-19, respectively (Fig. $2 d$; ref. 10). In addition, a HindIII fragment of approximately $15 \mathrm{~kb}$ is faintly, but specifically, detected in patient B13. None of the rearranged bands observed here were detected among 21 spouses who married into the families, or among 84 unrelated controls (data not shown); thus, it is highly unlikely that they represent polymorphic rearrangements. No changes in restriction patterns were found in samples from patient EUR21.1 (data not shown).
From the combined data, we conclude the heterozygous presence of three distinct large genomic deletions (Fig. $2 d$ ), one comprising exon 22 (in RUL5 and RUL105), one comprising exon 13 (in B20 and EUR9) and one comprising exons 13-16 (in B13). The finding that patient EUR9.5 carries the exon-13 deletion was unexpected, as we were unable to detect the corresponding RTPCR variant.

To further characterize these deletions, we used intronic amplimers to obtain PCR products from genomic DNA, specifically containing the deletion-junction fragments. Amplimers flanking exon 22 generated an aberrant genomic fragment in patients RUL5.6 and RUL105.1 (Fig. 3a), which turned out to contain a 510-bp deletion (Fig. 3c). This deletion starts in intron 21 and ends within the most upstream copy of three head-to-tail arranged Alu elements in intron 22 (Fig. $3 \mathrm{c}$ ). Similarly, amplimers flanking exon 13 generated a specific PCR product in patients B20 and EUR9.5 (Fig. $3 b$ ), which was shown to contain a 3,835-bp deletion. This deletion starts 112 bp downstream of an Alu element in intron 12 and ends in intron 13 in a region that shares very high homology with this element (Fig. $3 d$ ). The sizes of both deletions perfectly fit the observed restriction patterns (Fig. $2 d$ ). The 3 ' breakpoints of both deletions are closely flanked on either side by two 26-bp sequences that bear strong homology with the $A l u$ core sequence that is implied to stimulate recombination ${ }^{11}$.

In our total set of 170 research families, we had already found 21 
$a$

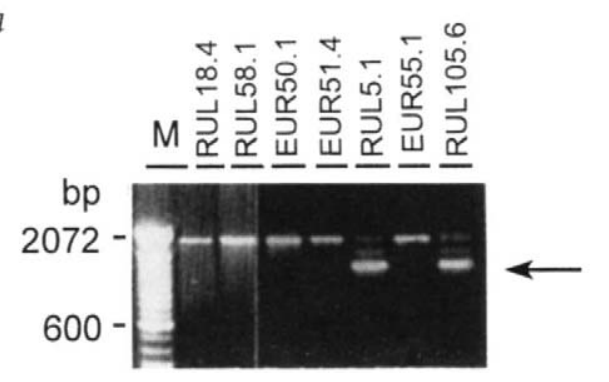

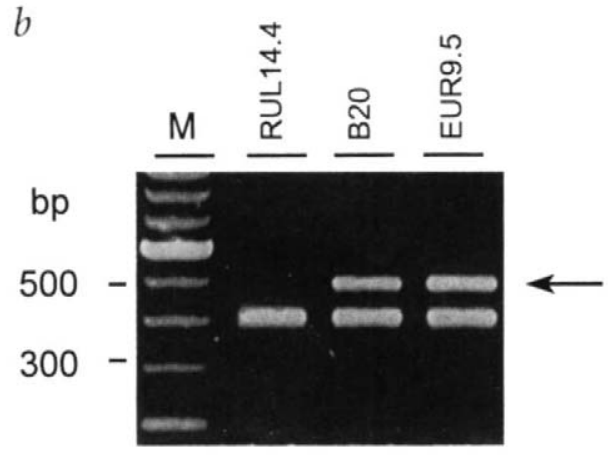

C
Fig. 3 PCR detection and sequence analysis of deletion-junction fragments. a, The 510-bp deletion containing exon 22 (arrow) is preferentially amplified relative to the wild-type allele. $\boldsymbol{b}$, The 3,835-bp deletion containing exon 13 (arrow). The wild-type allele of $4.4 \mathrm{~kb}$ was not amplified under these conditions. As an internal control for PCR efficiency, a fragment from exon 11 was co-amplified. c, Sequence of exon 22 (upper case) and its flanking intron sequence (lower case). Shaded box denotes the deleted 510 bp. Italicized sequence denotes the first 241 bp of an $A / u$ element. Numbers refer to nucleotide (nt) positions of the genomic sequence of BRCA1 (ref. 10). Bold sequences are imperfect copies of a common 26-bp recombinogenic core sequence ${ }^{11}$. d, Alignment of sequences of intron 12 (upper), the deletion-junction fragment (Jnctn, middle) and intron 13 (lower). Shaded box indicates the 10 bp where the recombination presumably took place that led to the exon 13 deletion. Nt 44481-44551 (intron 12) derive from an Alu element and share $85 \%$ identity with nt $48316-48386$ (intron 13). .

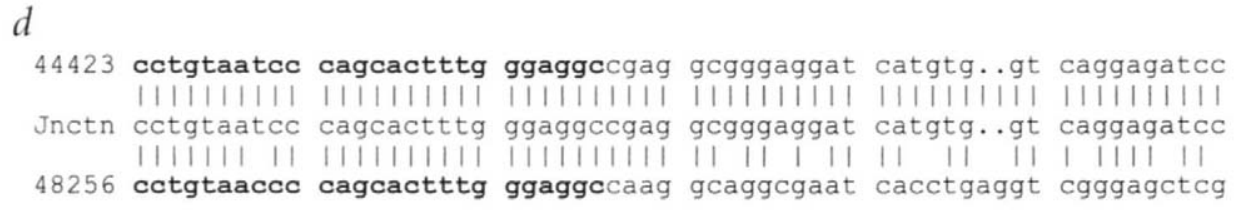

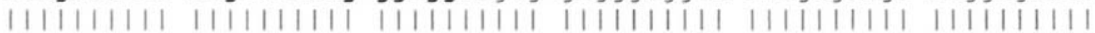
Jnctn cctgtaatcc cagcactttg ggaggccgag gcgggaggat catgtg..gt caggagatcc |111111 |1 |111111111 |111111111 |1 |1 | |1 |1 |1 |1 | |111 |1

48256 cctgtaaccc cagcactttg ggaggccaag gcaggcgaat cacctgaggt cgggagctcg

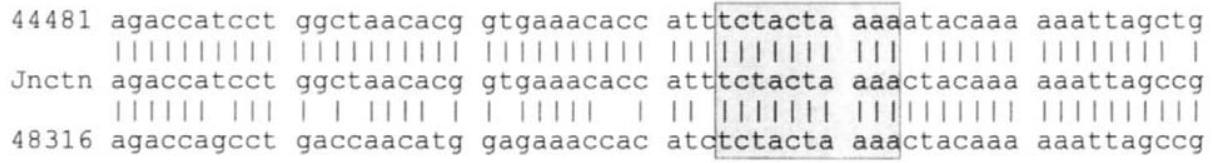

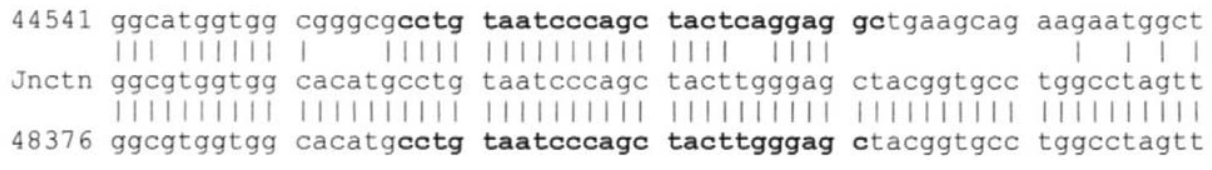

$B R C A 1$ and 9 BRCA2 mutations by PTT and PCR fragmentlength analysis ${ }^{4,7,8}$. Because strong founder effects have been shown for most recurrent Dutch $B R C A 1$ mutations ${ }^{4}$, we examined the remaining 137 breast-cancer research families for the occurrence of the 510- and 3,835-bp deletions. These were found in six and three additional families, respectively (Table 1). In all of these families, the deletion co-segregated with the disease. The rest were investigated for the presence of an SspI restriction site in intron 22, created by the IVS $22+5 \mathrm{G} \rightarrow \mathrm{A}$ mutation as observed in EUR21; none were observed (data not shown). Together with previous mutation screening results, the two deletions thus comprise $12 / 33$ $(36 \%)$ of all mutations in families which a $B R C A 1$ mutation has been detected. Furthermore, among the ten breast-cancer families predicted by linkage analysis 8 to carry mutations in $B R C A 1$, we have now detected a mutation in nine, three of which (33\%) carry one of the large deletions described here. A further indication of the frequency of the 510-bp deletion came from another set of samples derived from the Family Cancer Clinic in Amsterdam. A deletion of exon 22 had been detected independently at the cDNA level in 6 of 49 probands analysed, without evidence for the IVS22 $+5 \mathrm{G} \rightarrow$ A change. In a subsequent double-blind study exam- ining the genomic DNA of these samples, our PCR-based assay identified the 510-bp deletion in all six cases (data not shown).

We used three intragenic and two flanking markers to reconstruct the disease haplotype for each of the research families carrying either the 510 - or the $3,835-b p$ deletion (Table 1). Strong conservation of allele lengths was observed at the intragenic loci among the haplotypes carrying the same deletion, suggesting their descent from a common ancestor. The haplotype carrying the IVS22 $+5 \mathrm{G} \rightarrow \mathrm{A}$ mutation in EUR21 does indeed differ from the haplotype carrying the 510-bp deletion.

Our results are the first to show the importance of large genomic deletions in $B R C A 1$ among breast-cancer families from a defined Caucasian subpopulation. It has been proposed that the unusually high concentration of $A l u$ elements in the $B R C A 1$ intronic regions might render the gene particularly prone to intragenic recombination/deletion events ${ }^{10}$. A 1-kb Alu-mediated deletion affecting exon 17 of $B R C A 1$ was recently detected in a single large French breast-cancer family ${ }^{12}$. This lends further support to our hypothesis, and fuels the discussion of whether or not $B R C A 1$ is inactivated by somatic mutations in sporadic breast cancer ${ }^{13}$. Rather than small deletions and insertions, none of which have 
Table 1 - Disease-linked haplotypes of families with a recurrent deletion mutation

\begin{tabular}{|c|c|c|c|c|c|c|}
\hline Family & $\begin{array}{l}\text { Proband's } \\
\text { diagnosis' }\end{array}$ & \multicolumn{5}{|c|}{ Linked allele length ${ }^{b}$ at polymorphic marker (bp) } \\
\hline $\begin{array}{l}\text { 3,835-bp deletion: } \\
\text { B20 } \\
\text { EUR9 } \\
\text { EUR18 } \\
\text { EUR19 } \\
\text { EUR31 }\end{array}$ & $\begin{array}{c}\text { Br43 } \\
\text { Br28/Br46 } \\
\text { Ov54 } \\
\text { Ov38 } \\
\text { Ov36/Br43/Br49 } \\
\text { Allele frequency }\end{array}$ & $\begin{array}{c}169 / 167 \\
169 \\
169 \\
169 \\
169 \\
0.08\end{array}$ & $\begin{array}{c}151 / 145 \\
151 \\
151 \\
151 / 153 \\
151 \\
0.21\end{array}$ & $\begin{array}{c}122 / 128 \\
122 / 128 \\
122 \\
122 \\
122 \\
0.51\end{array}$ & $\begin{array}{c}151 / 159 \\
151 \\
151 \\
151 \\
151 \\
0.68\end{array}$ & $\begin{array}{c}133 / 169 \\
133 \\
133 \\
133 \\
133 \\
0.60\end{array}$ \\
\hline $\begin{array}{l}\text { 510-bp deletion: } \\
\text { RUL5 } \\
\text { RUL16 } \\
\text { RUL20 } \\
\text { RUL78 } \\
\text { RUL80 } \\
\text { RUL97 } \\
\text { RUL105 } \\
\text { EUR52 }\end{array}$ & $\begin{array}{c}\text { Br41 } \\
\text { Br41/Ov48 } \\
\text { Br64 } \\
\text { Br31 } \\
\text { Br41 } \\
\text { Br35/Br52 } \\
\text { Ov40 } \\
\text { Ov50 } \\
\text { Allele frequencyc }\end{array}$ & $\begin{array}{c}167 \\
167 \\
167 \\
167 \\
167 \\
167 / 169 \\
171 \\
167 \\
0.19\end{array}$ & $\begin{array}{c}155 \\
155 \\
155 \\
155 \\
155 \\
155 / 149 \\
155 \\
155 \\
0.09\end{array}$ & $\begin{array}{c}122 \\
122 \\
122 \\
122 \\
122 \\
122 / 125 \\
122 \\
122 \\
0.51\end{array}$ & $\begin{array}{c}151 \\
151 \\
151 \\
151 \\
151 \\
151 / 157 \\
151 \\
151 \\
0.68\end{array}$ & $\begin{array}{c}133 \\
133 \\
133 \\
133 \\
133 \\
133 / 157 \\
133 \\
133 \\
0.60\end{array}$ \\
\hline $\begin{array}{l}\text { IVS22 + } 5 G \rightarrow A: \\
\text { EUR21 }\end{array}$ & $\begin{array}{c}\text { Br41 } \\
\text { Allele frequencyc }\end{array}$ & $\begin{array}{l}167 \\
0.19\end{array}$ & $\begin{array}{l}149 \\
0.16\end{array}$ & $\begin{array}{l}125 \\
0.25\end{array}$ & $\begin{array}{l}151 \\
0.68\end{array}$ & $\begin{array}{l}155 \\
0.02\end{array}$ \\
\hline
\end{tabular}

a Br43 indicates that age of onset of breast cancer was 43 y; Ov54 indicates that age of onset of ovarian cancer was 54 y. ${ }^{b}$ When marker was uninformative to determine phasing, both alleles are listed. 'Of most commonly shared allele only, as measured among 168 unrelated Dutch control individuals 4 .

been reported to occur somatically in breast tumours ${ }^{14,15}$, Alumediated large genomic deletions might be a more common mechanism that inactivates $B R C A 1$ in sporadic breast cancer.

Because strong founder effects have been observed for many $B R C A 1$ mutations detected worldwide ${ }^{5,6}$, large deletions may constitute a substantial proportion of the mutation spectrum in certain ethnic subpopulations, as evidenced here for the Dutch. This could explain the discrepancy between the proportion of $B R C A I$ mutations predicted by linkage studies ${ }^{16,17}$ and the actual prevalence established by mutation analysis of breast-cancer families derived from a variety of ethnic backgrounds ${ }^{6,18-22}$. Although such deletions are detectable by RT-PCR analysis, the latter has not yet been widely used for mutation detection, and might be prone to false-negative results (for instance, as seen in the case of EUR9.5), either because the low abundance of BRCA1 mRNA in lymphocytes causes coincidental preferential amplification of the wild-type transcript (Fig. $1 b$ ) or because transcripts carrying exonic deletions are more unstable than the full-length transcript ${ }^{12}$. Our data clearly show that a comprehensive BRCA1 mutation test should include the examination of its genomic structure.

\section{Methods \\ Family ascertainment. Research families were ascertained either by the Netherlands Foundation for the Detection of Hereditary Tumors or by the Rotterdam Family Cancer Clinic. They all contain at least three first- degree relatives with either breast or ovarian cancer, at least one of them having received a diagnosis before 50 years of age. Of the 170 thus recruited, there were 48 families with at least four cases of breast cancer diagnosed before age 60 , in addition to any number of ovarian cancers ${ }^{4}$. Patients entered the study by application or physician referral to a family cancer clinic or clinical genetics department in either Amsterdam or Leiden. In general, these patients have an a priori chance of $10 \%$ or higher of being carriers of a BRCA1 mutation ${ }^{23}$.}

DNA and RNA isolation, reverse transcription and nested PCR. Isolation of genomic DNA and total RNA from fresh blood samples and preparation of first-strand cDNA by reverse transcription have been described ${ }^{7}$. For the RT-PCR analysis of exons 12-24, we used the following primers for the first PCR: forward (F), 5'-TCACAGTG CAGTGAATTGGAAG-3'; reverse (R), 5'-GTAGCCAGGACAGTAGAAGGACTG-3'. Nested primers were then used in a subsequent PCR to amplify exons 12-24 (F, 5'-GAAGAAA-
GAGGAACGGGCTTGG-3'; R, 5'-GGCCACTTTGTAAGCTCATTC-3'). For nested PCR of exons $20-24$, the same reverse primer was used in combination with the F: 5'-AACCACCAAGGTCCAAAGC-3'. PCR conditions were as described previously ${ }^{7}$.

Genomic PCR of the deletions spanning exons 13 or 22. A PCR reaction of $50 \mathrm{ml}$ typically contained $200 \mathrm{ng}$ of genomic DNA, 10-pmol primers, 0.75 U AmpliTaq Taq polymerase (Perkin Elmer/Cetus) and $5 \mathrm{ml}$ of either $\times 10 \mathrm{RM}$ buffer $(500 \mathrm{mM} \mathrm{KCl}, 100 \mathrm{mM}$ TRIS- $\mathrm{HCl} \mathrm{pH} \mathrm{8.4,} 25 \mathrm{mM} \mathrm{MgCl}$, $2 \mathrm{mg} / \mathrm{ml}$ BSA, $2 \mathrm{mM}$ dNTPs) for exon 13 or Optiprime buffer \#6 (Stratagene), supplemented with $0.1 \mathrm{mM}$ dNTPs for exon 22. For exon 13, the primers were (F) 5'-CAATGTGTTCCTGCCCTACT-3' and (R) 5-ACACTGGAAGACAACAGATATTAA-3', while an internal control fragment from exon 11 was co-amplified simultaneously (primers $F$, 5'-GTACAGTGAGCACAATTAGCCG-3'; R, 5'-TCTCAGAACAAACCTGAGATGC $\left.-3^{\prime}\right)$. This mixture was heated at $94^{\circ} \mathrm{C}$ for $1 \mathrm{~min}$, followed by 32 cycles of PCR $\left(1 \mathrm{~min}\right.$ at $94^{\circ} \mathrm{C}, 1.5 \mathrm{~min}$ at $56^{\circ} \mathrm{C}$ and $2 \mathrm{~min}$ at $\left.73^{\circ} \mathrm{C}\right)$ on a Perkin Elmer/Cetus DNA thermal cycler. For exon 22, the primers were (F) 5'-TCCCATTGAGAGGTCTTGCT-3' and (R) 5'-ACTGTGCTACTCAAGCACCA $-3^{\prime}$, After heating at $94^{\circ} \mathrm{C}$ for $5 \mathrm{~min}$, the PCR consisted of 35 cycles $\left(45 \mathrm{~s}\right.$ at $94^{\circ} \mathrm{C}, 1 \mathrm{~min}$ at $52^{\circ} \mathrm{C}$ and $2.5 \mathrm{~min}$ at $72^{\circ} \mathrm{C}$ ). All PCRs were concluded by an incubation at $73^{\circ} \mathrm{C}$ for $4 \mathrm{~min}$. The PCR products $(5 \mathrm{ml})$ were analysed on a $1.5 \%$ agarose gel.

Southern analysis. Genomic DNA $(5 \mu \mathrm{g})$ was digested with BglII or HindIII according to the supplier's protocols (Pharmacia). Agarose gels $(0.8 \%$ ) were run at $30 \mathrm{~V}$ for $16 \mathrm{~h}$ in TAE buffer ( $40 \mathrm{mM}$ Tris-HAc pH 8.3, 1 MM EDTA). Procedures for denaturing and transferring the separated DNA to nylon membranes (Hybond $\mathrm{N}^{+}$, Amersham) have been previously described ${ }^{24}$. As probes we used PCR products obtained from a clone containing the complete BRCA1 cDNA, and purified with the QIAquick PCR Purification Kit (Qiagen). Probe 11 (pl1) derives entirely from exon 11 and was obtained with the primers (F) 5'-GAAAAAAAAGTACAACCAAATGCC-3' and (R) 5'-AGCCCACTTCATTAGTACTGGAAC-3'. Probe 1424 (p1424) contains exons 14-24 and was obtained with the primers (F) 5'-TACCCTATAAGCCAGAATCCAGAA-3' and (R) $5^{\prime}-\mathrm{GGC}$ CACTTTGTAAGCTCATTC-3'. Purified fragments were labelled using the Megaprime DNA labelling system (Amersham) according to the supplier's protocols. Hybridizations were carried out overnight at $65^{\circ} \mathrm{C}$ in $125 \mathrm{mM} \mathrm{Na} \mathrm{HPO}_{4} \cdot 2 \mathrm{H}_{2} \mathrm{O}, 7 \%$ SDS, $10 \%$ PEG-6000, $1 \mathrm{mM}$ EDTA. Final washes were in $45 \mathrm{mM} \mathrm{NaCl}, 4.5 \mathrm{mM} \mathrm{Na}$ citrate $\mathrm{pH} 7.0,0.1 \% \mathrm{SDS}$, at $65^{\circ} \mathrm{C}$ for $30 \mathrm{~min}$. Filters were exposed overnight to a PhosphorImager screen (ImageQuant, Molecular Dynamics) and subsequently to Kodak X-Omat autoradiograms for 3 days at $-70^{\circ} \mathrm{C}$. 
Sequencing. Direct sequencing of PCR fragments was performed with M13-tailed and biotinylated primers used for PCR and subsequent solidphase sequencing with fluoresceinated primers on a Pharmacia A.L.F. Sequencer. Primer sequences have been published previously ${ }^{25}$.

Polymorphic marker analysis. Five polymorphic microsatellite markers located on chromosome $17 \mathrm{q}$, spanning an approximately 3 -cM interval including $B R C A 1$, were used in a radioactive $P C R$ under conditions described elsewhere ${ }^{4}$. D17S1323 has been mapped to intron 12 of BRCA1, $D 17 S 1322$ to intron 19 , and D17S855 to intron 20 (ref. 10). THRA1 is approximately $2 \mathrm{cM}$ proximal of $B R C A 1$, and $D 17 S 1327100 \mathrm{~kb}$ distal ${ }^{5,16}$ to $B R C A 1$. CEPH sample 1347 was used as a reference to estimate the size of alleles in base pairs.

1. Couch, F.J. et al. Mutations and polymorphisms in the familial early onset breast cancer (BRCA1) gene. Hum. Mutat. 8, 8-18 (1996).

2. Roa, B.B., Boyd, A.A., Volcik, K. \& Richards, C.S. Ashkenazi Jewish population frequencies for common mutations in BRCA1 and BRCA2. Nature Genet. 14, $185-187$ (1996).

3. Caligo, M.A. et al. BRCA1 germline mutational spectrum in italian families from Tuscany: a high frequency of novel mutations. Oncogene 13, 1483-1488 (1996).

4. Peelen, T. et al. A high proportion of novel mutations in BRCA1 with strong founder effects among Dutch and Belgian hereditary breast and ovarian cancer founder effects among Dutch and Belgian hereditary.

5. Neuhausen, S.L. et al. Haplotype and phenotype analysis of six recurrent BRCA1 mutations in 61 families: results of an international study. Am. J. Hum. Genet. 58, 271-280 (1996)

6. Szabo, C.I. \& King, M.-C. Population genetics of BRCA1 and BRCA2. Am. J. Hum. Genet. 60, 1013-1020 (1997)

7. Hogervorst, F.B.L. et al. Rapid detection of BRCA1 mutations by the protein truncation test. Nature Genet. 10, 208-212 (1995).

8. Peelen, T. et al. The majority of 22 Dutch high-risk breast cancer families are due to either BRCA1 or BRCA2. Eur. J. Hum. Genet. 4, 225-230 (1996).

9. Shapiro, M.B. \& Senapathy, P. RNA splice junctions of different classes of eukaryotes: sequence statistics and functional implications in gene expression. Nucleic Acids Res. 15, 7155-7174 (1997).

10. Smith, T.M. et al. Complete genomic sequence and analysis of $117 \mathrm{~kb}$ of human DNA containing the gene BRCA1. Genome Res. 6, 1029-1049 (1996).

11. Rudiger, N.S., Gregersen, N. \& Kieliand-Brandt, M.C. One short well conserved region of Alu-sequences is involved in human gene rearrangements and has homology with prokaryotic chi. Nucleic Acids Res. 23, 256-260 (1995).

12. Puget, N. et al. A 1 -kb Alu-mediated germ-line deletion removing BRCA1 exon 17. Cancer Res. 57, 828-831 (1997).

13. Szabo, C.I. \& King, M.-C. Inherited breast and ovarian cancer. Hum. Mol. Genet. 4,

\section{Acknowledgements}

We thank the families for their cooperation and $I$, van Leeuwen for ascertainment and taking blood samples. G. Brink is acknowledged for his expert technical assistance. A plasmid clone containing the full-length $B R C A 1 \mathrm{cDNA}$ was the gift of $B$. Weber and $F$. Couch. This work was supported by the Dutch Cancer Society (grant RUL95-1035) and the Nijbakker-Morra Foundation.

Received 7 May; accepted 17 September 1997

1811-1817 (1995)

14. Futreal, P.A. et al. BRCA1 mutations in primary breast and ovarian carcinomas. Science 266, 120-122 (1994)

15. Katagiri, T. et al. Mutations in the BRCA1 gene in Japanese breast cancer patients, Hum. Mutat. 7, 334-339 (1996)

16. Easton, D., Bishop, D., Ford, D. Crockford, G. \& the Breast Cancer Linkage Consortium. Genetic linkage analysis in familial breast and ovarian cancer: results from 214 families. Am. J. Hum. Genet. 52, 678-701 (1993).

17. Narod, 5 . et al. An evaluation of genetic heterogeneity in 145 breast-ovarian cancer families. Am. J. Hum. Genet. 56, 254-264 (1995).

18. Castilla, L. et al. Mutations in the BRCA1 gene in families with early-onset breast and ovarian cancer. Nature Genet. 8, 387-391 (1994).

19. Inove, R. et al. Germline mutation of 8RCA1 in Japanese breast cancer families. Cancer Res. 55, 3521-3524 (1995).

20. Durocher, F. et al. Mutation analysis of the BRCA1 gene in 23 families with cases of cancer of the breast, ovary, and multiple other sites. J. Med. Genet. 33. 814-819 (1996)

21. Xu, C.F. et al. Mutations and alternative splicing of the BRCA1 gene in UK breast/ovarian cancer families. Genes Chrom. Cancer 18, 102-110 (1997).

22. Hamann, U., Brauch, H., Garvin, A.M., Bastert, G. \& Scott, R.J. German family study on hereditary breast and/or ovarian cancer: germline mutation analysis of the BRCA1 gene. Genes Chrom. Cancer 18, 126-132 (1997).

23. Shattuck-Eidens, D. et al. A collaborative survey of 80 mutations in the BRCAI breast and ovarian cancer susceptibility gene: implications for presymptomatic testing and screening. JAMA 273, 535-541 (1995).

24. Devilee, P. et al. At least four different chromosomal regions are involved in loss of heterozygosity in human breast carcinoma. Genomics 5, 554-560 (1989).

25. Friedman, $L$. et al. Confirmation of BRCA1 by analysis of germline mutations linked to breast and ovarian cancer in ten families. Nature Genet. 8, 399-404 (1994). 